\title{
Perfil de gastos com o tratamento da Artrite Reumatoide para pacientes do Sistema Único de Saúde em Minas Gerais, Brasil, de 2008 a 2013
}

\author{
Profile of spending on the treatment of rheumatoid arthritis \\ for patients of the Unified Health System \\ in the state of Minas Gerais - 2008-2013
}

Grazielle Dias da Silva ${ }^{1}$

Eli Iola Gurgel Andrade ${ }^{2}$

Mariângela Leal Cherchiglia ${ }^{2}$

Alessandra Maciel Almeida ${ }^{3}$

Augusto Afonso Guerra Júnior ${ }^{3}$

Francisco de Assis Acurcio ${ }^{3}$

${ }^{1}$ Fundação Hemominas, Secretaria de Estado da Saúde de Minas Gerais. R. Grão Pará $882 / 7^{\circ}$, Santa Efigênia. 30150-341 Belo Horizonte MG Brasil. grazidiassilva@gmail.com

${ }^{2}$ Faculdade de Medicina, Universidade Federal de Minas Gerais (UFMG). Belo Horizonte MG Brasil.

${ }^{3}$ Faculdade de Farmácia, UFMG. Belo Horizonte MG Brasil.

\begin{abstract}
Rheumatoid arthritis ( $R A)$ is a chronic condition that affects about $1 \%$ of the adult population. In a historical cohort of Minas Gerais State, 11,573 RA patients registered in the Outpatient Information System (SIA) between 2008 and 2013 were identified. For this study we adopted the public funding body's perspective and the values were adjusted by the national inflation index (IPCA) of December 2015. Etanercept was the most expensive treatment. The mean cohort age was 52 years old and most of the patients were women. Multiple regression analysis indicated a negative association between higher expenditure and age, female sex, and diagnosis at entry in the cohort and positive association between high expenditure and the Human Development Index (HDI) of the municipality and use of tumor necrosis factor agents. This study identified the factors that have an impact on $R A$ drug treatment expenditure. Also, we showed that methods that enable extracting demographic and expenditure data of administrative information systems may represent important tools in the construction of economic studies to subsidize economic health evaluations, especially from the standpoint of the managers.
\end{abstract}

Key words Rheumatoid arthritis, Pharmaceutical economics, Unified Health System, Drugs from the Specialized Component of Pharmaceutical Care (CEAF)
Resumo A artrite reumatoide (AR) é uma doença crônica que afeta cerca de $1 \%$ da população adulta. No estudo de coorte histórica de pacientes de Minas Gerais, registrados no Sistema de Informações Ambulatoriais (SIA), em 2008-2013, foram identificados 11.573 indivíduos. A perspectiva foi a do financiador público e os valores observados como gastos do Sistema Único de Saúde (SUS) foram ajustados pelo Índice Nacional de Preços ao Consumidor Amplo (IPCA), de dezembro de 2015. O Etanercept foi o tratamento mais caro. A análise múltipla mostrou uma relação negativa entre o aumento das despesas e idade, sexo feminino e diagnóstico de entrada na coorte, e relação positiva para as variáveis Índice de Desenvolvimento Humano Municipal (IDH-M) e o uso de medicamentos bloqueadores do fator de necrose tumoral (ANTI-TNF). Este estudo identificou os fatores que têm impacto sobre o gasto com o tratamento medicamentoso da AR. Também apontou que métodos que permitem extrair dados demográficos e de gastos de sistemas de informação administrativos podem ser ferramentas importantes na construção de estudos econômicos capazes de subsidiar as avaliações econômicas de saúde, especialmente do ponto de vista da gestão.

Palavras-chave Artrite reumatoide, Farmacoeconomia, Sistema Único de Saúde, Medicamentos do omponente Especializado da Assistência Farmacêutica (CEAF) 


\section{Introdução}

A artrite reumatoide (AR) é uma doença inflamatória sistêmica, autoimune, que pode levar à destruição cartilaginosa e óssea ${ }^{1,2}$. É uma condição crônica e progressiva que afeta cerca de $0,5 \%$ a $1 \%$ da população mundial adulta, preferencialmente mulheres e indivíduos na faixa etária de 40 a 60 anos $^{1-3}$. A prevalência na população brasileira foi estimada entre 0,2 e $1 \% \%^{4,5}$. Alamanos et al. (2006) observaram que diferenças nos métodos de identificação e registros de casos, bem como diferenças na prática médica, no acesso aos cuidados de saúde e a variabilidade na prevalência de riscos ambientais e fatores genéticos, podem influenciar nas taxas observadas nos estudos avaliados ${ }^{6}$.

Os primeiros doze meses da doença são considerados uma "janela de oportunidade" e pacientes tratados neste período têm melhores prognósticos ${ }^{1-3,7}$. No Brasil, os medicamentos são fornecidos pelo Sistema Único de Saúde (SUS) por meio do Componente Especializado da Assistência Farmacêutica (CEAF), anteriormente denominado Programa Nacional de Medicamentos de Dispensação Excepcional/Alto Custo (PMAC). Este componente inclui medicamentos considerados de alto custo, que são aqueles de alto valor agregado ou, que pela cronicidade do tratamento se tornam excessivamente caros para serem financiados pela população8.

Estudo avaliando os gastos de todos os pacientes cadastrados no PMAC, iniciando tratamento entre 2000 e 2004, constatou que indivíduos utilizando infliximabe (o primeiro medicamento biológico disponibilizado pelo CEAF para o tratamento da AR) apresentaram a segunda maior média de gasto no período ${ }^{9}$. Costa et al. ${ }^{10}$, ao avaliar os gastos com este medicamento no CEAF, indicaram diferença significativa nos valores despendidos conforme sexo, diagnóstico e macrorregião de residência do paciente. Também houve diferença significativa nos gastos analisados neste estudo para seguimentos terapêuticos distintos ${ }^{10}$. O tempo de tratamento também pode ser um fator importante na variabilidade dos gastos com o tratamento da AR, uma vez que tratamentos mais caros podem substituir os tratamentos tradicionais e de menor valor agregado na busca por melhores resultados terapêuticos ${ }^{11}$.

O CEAF é executado e gerenciado pelos estados e financiado em conjunto com o Ministério da Saúde por meio do Fundo de Ações Estratégicas e Compensação (FAEC). A aquisição dos medicamentos que compõem o CEAF pode ser realizada de forma centralizada no Ministério da Saúde ou de forma descentralizada nos estados. Para este último caso, o financiamento por parte do Ministério da Saúde é realizado por meio dos valores estabelecidos para a Autorização de Procedimentos de Alta Complexidade/Custo (APAC), que são então utilizados como parâmetro para ressarcimento aos estados dos valores despendidos na aquisição dos medicamentos. Os valores das APACs integram o conjunto de dados do Sistema de Informações Ambulatoriais $(\text { SIA })^{12}$. Desta forma, os valores apresentados no SIA/SUS para o CEAF incluem apenas os gastos despendidos pelo Ministério da Saúde com o ressarcimento aos estados, podendo ou não refletir o custo real de compra descentralizada do medicamento. Cabe salientar que melhorias nas ferramentas de gestão do CEAF, ao longo do tempo, também podem promover variabilidade nos gastos do SUS com estes medicamentos, na medida em aumentam a qualidade dos registros.

Assim, com o intuito de contribuir para o conhecimento acerca do tratamento medicamentoso desta doença, este artigo tem por objetivo apresentar uma avaliação do perfil dos gastos com o tratamento da Artrite Reumatoide utilizado por pacientes do SUS em Minas Gerais (MG), no período de janeiro de 2008 a setembro de 2013.

\section{Métodos}

Este estudo é parte integrante do Projeto de Pesquisa "Avaliação da efetividade e segurança dos anticorpos monoclonais adalimumabe, infliximabe, etanercepte e rituximabe utilizados no tratamento da artrite reumatoide, artrite psoriática e espondilite anquilosante, Brasil e Minas Gerais", aprovado pelo Comitê de Ética em Pesquisa da Universidade Federal de Minas Gerais.

\section{Tipo de estudo}

Foi realizado um estudo de coorte retrospectiva composta por pacientes que utilizaram medicamentos de alto custo para o tratamento da AR no estado de MG, no período de janeiro de 2008 a setembro de 2013. O Protocolo Clínico e Diretrizes Terapêuticas (PCDT) da AR estabelece um prazo de 3 a 6 meses para verificar falha terapêutica do tratamento ${ }^{2}$. Desta forma foram incluídos na coorte os pacientes que completaram o período mínimo de seis meses de acompanhamento. 
Utilizou-se a base de dados do SIA do SUS/ MG pareada pelo método de linkage determinístico-probabilístico conforme descrito por Cherchiglia et al. ${ }^{13}$. Para isso utilizou-se software Freely Extensible Biomedical Record Linkage - Febrl, versão $0.3^{14}$. Segundo os autores, o linkage teve como objetivo encontrar os registros de um mesmo paciente nos diferentes arquivos da base de dados para unificá-los. O método de linkage determinístico foi aplicado aos identificadores únicos confiáveis e/ou com base em comparação de múltiplos identificadores. Já o método de linkage probabilístico foi aplicado nos arquivos que apresentavam problemas de consistência, erros ou informações não declaradas ${ }^{13}$.

Para identificação dos indivíduos da coorte de MG foram considerados os Códigos Internacionais de Doença (CID), bem como os códigos de procedimentos definidos pelo PCDT (2006) 2 da AR. Os medicamentos incluídos na análise foram Adalimumabe (ADA); Etanercepte (ETA); Infliximabe (INF); Metotrexato (MTX); Azatioprina (AZA); Ciclosporina (CIC); Cloroquina (CLO); Hidroxicloroquina (HID); Leflunomida (LEF) e Sulfassalazina (SUL).

\section{Avaliação dos gastos}

A avaliação dos gastos foi realizada considerando a perspectiva do financiador público e foi limitada aos gastos ambulatoriais de medicamentos registrados no SIA/SUS. Para precificação dos medicamentos, utilizou-se a média dos valores registrados no sítio eletrônico do Banco de Preços em Saúde do Ministério da Saúde (BPS) para cada ano de acompanhamento (Tabela 1). A pesquisa de preços foi realizada em março de 2016 e os valores foram atualizados utilizando-se o Índice de Preços ao Consumidor Amplo (IPCA) do Instituto Brasileiro de Geografia e Estatística (IBGE), para dezembro de 2015. Para os medicamentos que não apresentavam registro de preços no BPS, foram utilizados os valores registrados como aprovados no sítio eletrônico do DATASUS, para o cálculo das médias dos valores unitários. Considerando que os dados de gastos foram extraídos de uma base de dados administrativa que não registra todos os custos envolvidos com o tratamento, assumiu-se os gastos registrados no SIA/SUS como proxy dos custos do tratamento da AR para análise dos dados. Na perspectiva adotada, gasto é definido como despesa com bens ou serviços adquiridos, enquanto custo compreende o valor de todos os recursos gastos na produção de um bem ou serviço.
Desta forma, os gastos foram contabilizados para cada indivíduo e avaliados como gasto médio mensal (GMM) para cada esquema terapêutico observado no intervalo de acompanhamento da coorte. A fim de sintetizar a apresentação dos resultados, os medicamentos foram classificados em quatro categorias conforme seu mecanismo de ação farmacológica: 1.Medicamentos Modificadores do Curso da Doença Sintéticos, exceto MTX - Outros MMCD sintéticos (CLO, HID, LEF e SUL); 2.METOTREXATO - MTX; 3.Bloqueadores do fator de necrose tumoral associados ou não aos outros MMCD sintéticos ou MTX - Anti-TNF ( \pm MMCD ou MTX) (ADA, ETA e INF) e, 4.Imunossupressores - IMUNO (AZA e CIC).

\section{Análise estatística}

A estatística descritiva dos dados coletados foi realizada por meio de medidas de tendência central e de dispersão para as variáveis contínuas e distribuições de frequências para as variáveis categóricas. Com a finalidade de melhor representar, nos modelos estatísticos adotados, as características sociodemográficas dos municípios de residência dos indivíduos incluídos na coorte, utilizou-se o IDH-M. O índice para cada município foi obtido no sítio eletrônico do IBGE ${ }^{15}$.

Para a identificação dos fatores que influenciaram o GMM com medicamentos no período estudado, realizaram-se análises de regressão linear bivariada e múltipla. As variáveis independentes incluídas na análise bivariada foram idade, sexo, tempo de acompanhamento, ano de entrada na coorte, CID identificado no início do acompanhamento, medicamento de início de tratamento e IDH-M do município de residência identificado no início do acompanhamento. O modelo final da regressão linear múltipla incluiu as variáveis independentes com resultado estatisticamente significativo a um nível de 5\% na análise de regressão linear bivariada. Foram realizados a análise dos gráficos de resíduos e o teste de Breusch-Pagan para testar a homoscedasticidade.

\section{Resultados}

A coorte de MG incluiu 11.573 indivíduos. Destes, a maioria $(81,3 \%)$ era do sexo feminino com idade média de 52 ( \pm 15 ) anos (Tabela 2). Os diagnósticos mais frequentes observados no início do acompanhamento foram "Outras artrites reumatoides soro-positivas" (43,9\%) e "Síndro- 
Tabela 1. Média ponderada de valores unitários para os medicamentos utilizados pelos indivíduos da Coorte calculada a partir de dados registrados no sítio eletrônico do Banco de Preços em Saúde (Valores em R\$ atualizados para 12/2015*)

\begin{tabular}{|c|c|c|c|c|c|c|c|}
\hline \multirow{3}{*}{ Ativo } & \multirow{3}{*}{$\begin{array}{l}\text { Unidade } \\
\text { de compra }\end{array}$} & \multicolumn{6}{|c|}{ Ano } \\
\hline & & \multicolumn{2}{|c|}{2008} & \multicolumn{2}{|c|}{2009} & \multicolumn{2}{|c|}{2010} \\
\hline & & MP & DP & MP & DP & MP & DP \\
\hline Adalimumabe $40 \mathrm{mg}$ & Seringa & $2.887,19$ & 266,47 & $2.633,62$ & 260,36 & $2.999,12$ & 194,09 \\
\hline Azatioprina 50mg & Comprimido & 1,17 & 0,50 & 1,45 & 0,53 & 0,76 & 0,53 \\
\hline Ciclosporina $25 \mathrm{mg}$ & Cápsula & 1,33 & 0,39 & 0,86 & 0,55 & 0,56 & 0,44 \\
\hline Ciclosporina 50mg & Cápsula & 2,95 & 0,58 & 1,71 & 1,01 & 0,95 & 0,90 \\
\hline Ciclosporina 100mg & Cápsula & 3,26 & 2,02 & 2,51 & 0,29 & 3,66 & 1,91 \\
\hline $\begin{array}{l}\text { Ciclosporina } 100 \mathrm{mg} / \mathrm{mL} \\
\text { Solução Oral }\end{array}$ & Frasco $50 \mathrm{ml}$ & 196,10 & 78,84 & 168,80 & 65,20 & 240,43 & 83,02 \\
\hline Cloroquina 150mg & Comprimido & 0,22 & - & 0,18 & 0,23 & 0,32 & 0,17 \\
\hline Etanercepte $25 \mathrm{mg}$ & Frasco ampola & 820,97 & 2,92 & 791,28 & 5,65 & 766,80 & 89,37 \\
\hline Etanercepte 50mg & Frasco ampola & NR & NR & $1.583,88$ & 39,50 & $1.583,32$ & 123,39 \\
\hline Hidroxicloroquina 400mg & Comprimido & 1,41 & 0,14 & 1,37 & 0,06 & 1,31 & 0,37 \\
\hline Infliximabe $10 \mathrm{mg} / \mathrm{mL}$ & Frasco ampola & $2.789,54$ & 182,50 & $2.636,11$ & 238,53 & $2.619,78$ & 221,76 \\
\hline Leflunomida 20mg & Comprimido & 8,93 & 0,04 & 8,46 & 0,90 & 8,08 & 0,66 \\
\hline Metotrexato 25mg & Ampola $2 \mathrm{~mL}$ & NR & NR & 7,32 & 0,85 & 7,09 & 0,66 \\
\hline Metotrexato 2,5mg & Comprimido & 0,85 & 0,14 & 0,82 & 0,06 & 0,79 & 0,08 \\
\hline Sulfassalazina $500 \mathrm{mg}$ & Comprimido & 0,71 & 0,02 & 0,68 & 0,23 & 0,71 & 0,23 \\
\hline \multirow[t]{2}{*}{ Ativo } & \multirow[t]{2}{*}{$\begin{array}{l}\text { Unidade } \\
\text { de compra }\end{array}$} & \multicolumn{2}{|c|}{2011} & \multicolumn{2}{|c|}{2012} & \multicolumn{2}{|c|}{2013} \\
\hline & & MP & DP & MP & DP & MP & DP \\
\hline Adalimumabe $40 \mathrm{mg}$ & Seringa & $2.438,78$ & 264,29 & $2.458,55$ & - & $2.339,98$ & 9,14 \\
\hline Azatioprina 50mg & Comprimido & 1,20 & 0,49 & 0,53 & 0,58 & 1,11 & 0,18 \\
\hline Ciclosporina $25 \mathrm{mg}$ & Cápsula & 1,07 & 0,56 & 0,60 & 0,23 & 1,01 & 0,46 \\
\hline Ciclosporina 50mg & Cápsula & 0,92 & 1,19 & 0,93 & 0,24 & 0,51 & - \\
\hline Ciclosporina 100mg & Cápsula & 1,22 & - & 5,07 & - & 0,79 & - \\
\hline $\begin{array}{l}\text { Ciclosporina } 100 \mathrm{mg} / \mathrm{mL} \\
\text { Solução Oral }\end{array}$ & Frasco $50 \mathrm{ml}$ & 227,17 & 72,04 & 193,95 & 45,10 & NR & NR \\
\hline Cloroquina 150mg & Comprimido & 0,29 & 1,30 & NR & NR & 0,42 & - \\
\hline Etanercepte $25 \mathrm{mg}$ & Frasco ampola & 753,04 & 16,47 & 738,95 & 1,33 & 697,88 & 7,13 \\
\hline Etanercepte 50mg & Frasco ampola & $1.522,93$ & 10,88 & $1.414,39$ & 38,05 & $1.370,56$ & - \\
\hline Hidroxicloroquina 400mg & Comprimido & 1,39 & 0,35 & 1,17 & 0,68 & 1,09 & - \\
\hline Infliximabe $10 \mathrm{mg} / \mathrm{mL}$ & Frasco ampola & $2.764,72$ & - & $2.461,47$ & 212,31 & $2.094,91$ & - \\
\hline Leflunomida 20mg & Comprimido & 7,66 & 1,93 & 7,10 & 0,81 & 6,77 & - \\
\hline Metotrexato $25 \mathrm{mg}$ & Ampola $2 \mathrm{~mL}$ & 13,02 & 5,49 & 6,35 & - & 6,35 & - \\
\hline Metotrexato 2,5mg & Comprimido & 0,79 & 0,25 & 0,79 & 0,09 & 0,77 & 0,08 \\
\hline Sulfassalazina $500 \mathrm{mg}$ & Comprimido & 0,62 & 0,21 & 0,58 & 0,00 & 0,56 & 0,02 \\
\hline
\end{tabular}

DP = Desvio Padrão; MP = Média Ponderada; NR = Não Registrado. * utilizando-se o Índice de Preços ao Consumidor Amplo (IPCA), IBGE.

me de Felty" (25,2\%). A maioria dos pacientes iniciou o acompanhamento na coorte em uso de Outros MMCD sintéticos (66,8\%) (Tabela 2). Em relação à distribuição dos pacientes confor- me o IDH-M do município de residência no primeiro registro de acompanhamento, observou-se que $53,1 \%$ dos pacientes residiam em municípios com IDH-M até 0,80 . 
Tabela 2. Características dos pacientes que fizeram uso de medicamentos de alto custo para o tratamento da Artrite Reumatóide no Sistema Único de Saúde de Minas de Gerais, no período de janeiro de 2008 a setembro de $2013(\mathrm{~N}=11.573)$.

\begin{tabular}{|c|c|c|}
\hline Características dos pacientes & $\mathbf{N}$ & $\%$ \\
\hline \multicolumn{3}{|l|}{ Idade $^{*}$} \\
\hline Média ( $\pm \mathrm{DP})$ & $51,7( \pm 14,6)$ & \\
\hline \multicolumn{3}{|l|}{ Sexo } \\
\hline $\mathrm{F}$ & 9.412 & 81,3 \\
\hline M & 2.161 & 18,7 \\
\hline \multicolumn{3}{|l|}{ Ano de início } \\
\hline 2008 & 2.641 & 22,8 \\
\hline 2009 & 1.222 & 10,6 \\
\hline 2010 & 1.095 & 9,5 \\
\hline 2011 & 4.120 & 35,6 \\
\hline 2012 & 2.024 & 17,5 \\
\hline 2013 & 471 & 4,1 \\
\hline \multicolumn{3}{|l|}{ Diagnóstico* } \\
\hline Outras artrites reumatóides soro-positivas (M05.8) & 5.078 & 43,9 \\
\hline Síndrome de Felty (M05.0) & 2.916 & 25,2 \\
\hline Artrite reumatóide soro-negativa (M06.0) & 1.769 & 15,3 \\
\hline Artrite reumatóide com comprometimento de outros órgãos e sistemas (M05.3) & 887 & 7,7 \\
\hline Outros & 923 & 8,0 \\
\hline \multicolumn{3}{|l|}{ Medicamento* } \\
\hline Outros MMCD sintéticos & 7.726 & 66,8 \\
\hline Anti-TNF ( \pm MMCD ou MTX) & 2.176 & 18,8 \\
\hline Metotrexato & 1.555 & 13,4 \\
\hline Imunossupressores & 116 & 1,0 \\
\hline \multicolumn{3}{|l|}{ IDH-M ${ }^{*}$} \\
\hline $0,00-0,77$ & 2.857 & 24,7 \\
\hline $0,78-0,80$ & 3.290 & 28,4 \\
\hline $0,81-0,83$ & 2.652 & 22,9 \\
\hline 0,84 ou mais & 2.774 & 24,0 \\
\hline
\end{tabular}

* Variável observada no primeiro registro do indivíduo na coorte. $\mathrm{MMCD}=$ Medicamentos Modificadores do Curso da Doença; Anti-TNF $=$ Anti-tumor necrosis fator; MTX = Metotrexato.

A maior parte dos pacientes permaneceu durante todo o período de acompanhamento no tratamento com o medicamento identificado no primeiro registro. Quando a mudança de tratamento foi identificada, observou-se que houve alteração no GMM. O tratamento dos pacientes que iniciaram o acompanhamento em uso de MTX (11\%) foi o de menor GMM no período de análise $(\mathrm{p}<0,001)$. Este gasto aumentou de forma significativa quando um Anti-TNF foi introduzido ( $p<0,001)$. Resultados semelhantes foram observados para os pacientes que iniciaram o acompanhamento em uso de outros MMCD sintéticos $(\mathrm{p}<0,001)$ (Tabela 3$)$.

Dos pacientes que iniciaram o tratamento com ADA ( \pm MMCD ou MTX) (9\%), 12\% mudaram para outro esquema terapêutico: MMCD ou MTX em monoterapia ou outro Anti-TNF ( \pm MMCD ou MTX). A mudança para um dos MMCD sintéticos resultou em um decréscimo no GMM em comparação à manutenção do tratamento inicial e em um acréscimo no GMM quando a mudança foi para outro Anti-TNF ( \pm MMCD ou MTX) (p $<0,001$ ). A mesma tendência foi observada para os pacientes que iniciaram o tratamento com INF ( \pm MMCD ou MTX) $(3 \%)(\mathrm{p}<0,001)$. O tratamento dos pacientes que iniciaram o acompanhamento em uso de ETA ( \pm MMCD ou MTX) (7\%) foi o de maior GMM no período de análise $(\mathrm{p}<$ 0,001 ). Para estes pacientes, tanto a mudança para um dos MMDC sintéticos quanto para outro Anti-TNF resultou em queda no GMM ( $p<0,001)$. 
Achados interessantes em relação ao tempo de acompanhamento também podem ser observados na Tabela 3. No grupo dos Anti-TNF observou-se um maior tempo médio de acompanhamento para o INF $(\mathrm{p}<0,001)$. Não houve diferença estatisticamente significativa na comparação entre os tempos médios de acompanhamento para pacientes tratados com ETA $( \pm$ MMCD ou MTX) e ADA ( \pm MMCD ou MTX). Entre os MMCD sintéticos (MMCD ou MTX), o MTX apresentou o menor tempo médio de acompanhamento $(\mathrm{p}>0,001)$. Na comparação com os Anti-TNF, os MMCD sintéticos (MTX ou outros) só não apresentaram maior tempo médio de acompanhamento que o INF ( $\mathrm{p}<0,05)$. O tempo médio de acompanhamento deste me- dicamento também foi maior que o observado para os IMUNO ( $\mathrm{p}>0,05)$. Esta tendência não foi observada para as comparações de tempo médio de acompanhamento entre os IMUNO e ADA ( $<<0,001)$, ETA $(\mathrm{p}<0,001)$, MMCD (p $<0,001)$ e MTX ( $p>0,05)$. Para os grupos que iniciaram o tratamento com MMCD sintéticos (MMCD ou MTX) ou com IMUNO, o tempo médio de acompanhamento aumentou com a associação de um Anti-TNF ( $p<0,001)$. Nos grupos que iniciaram o tratamento com Anti-TNF, o tempo médio de acompanhamento aumentou com a troca por outro Anti-TNF ( $\mathrm{p}<0,001)$.

A Tabela 4 apresenta os resultados da regressão linear múltipla. O modelo final é capaz de explicar em torno de $67 \%$ da variabilidade

Tabela 3. Descrição dos gastos médios mensais ambulatoriais por seguimento terapêutico - janeiro de 2008 a setembro de 2013 (valores atualizados para dezembro de 2015 conforme IPCA).

\begin{tabular}{|c|c|c|c|c|}
\hline Seguimento Terapêutico & $\mathbf{N}$ & $\%$ & $\begin{array}{l}\text { Tempo médio de } \\
\text { acompanhamento } \\
\text { (meses) }\end{array}$ & $\begin{array}{l}\text { Gasto médio } \\
\text { Mensal (R\$) }\end{array}$ \\
\hline Iniciaram com ADA & 990 & 9 & $26,72( \pm 14,20)$ & $2.330,59$ \\
\hline ADA ( \pm MMCD ou MTX) & 870 & 88 & $26,18( \pm 14,24)$ & $2.339,61$ \\
\hline ADA ( \pm MMCD ou MTX)-MMCD ou MTX & 58 & 6 & $28,41( \pm 13,92)$ & $1.135,44$ \\
\hline ADA ( \pm MMCD ou MTX)-Outro Anti-TNF ( \pm MMCD ou MTX) & 62 & 6 & $32,65( \pm 12,46)$ & $3.321,99$ \\
\hline Iniciaram com ETA & 866 & 7 & $27,04( \pm 14,34)$ & $4.434,76$ \\
\hline ETA ( \pm MMCD ou MTX) & 753 & 87 & $25,62( \pm 13,47)$ & $4.736,39$ \\
\hline ETA ( \pm MMCD ou MTX)-MMCD ou MTX & 59 & 7 & $32,94( \pm 15,58)$ & $2.027,06$ \\
\hline ETA ( \pm MMCD ou MTX)-Outro Anti-TNF ( \pm MMCD ou MTX) & 54 & 6 & $41,49( \pm 15,68)$ & $2.859,31$ \\
\hline Iniciaram com INF & 369 & 3 & $39,43( \pm 20,63)$ & $2.138,72$ \\
\hline INF ( \pm MMCD ou MTX) & 254 & 69 & $33,11( \pm 17,75)$ & $2.038,74$ \\
\hline INF ( \pm MMCD ou MTX)-Outro Anti-TNF ( \pm MMCD ou MTX) & 96 & 26 & $44,30( \pm 22,24)$ & $2.681,40$ \\
\hline INF ( \pm MMCD ou MTX)-MMCD ou MTX & 19 & 5 & $55,17( \pm 18,83)$ & 733,28 \\
\hline Iniciaram com MTX & 1.266 & 11 & $28,91( \pm 17,49)$ & 173,29 \\
\hline MTX & 765 & 60 & $26,16( \pm 16,63)$ & 21,22 \\
\hline MTX-MMCD & 373 & 29 & $20,48( \pm 13,82)$ & 79,16 \\
\hline MTX-Anti-TNF ( \pm MMCD ou MTX) & 104 & 8 & $34,11( \pm 16,11)$ & $1.650,76$ \\
\hline MTX+MMCD & 24 & 2 & $33,63( \pm 18,39)$ & 81,36 \\
\hline Iniciaram com MMCD sintéticos exceto MTX & 6.837 & 59 & $34,34( \pm 19,14)$ & 425,04 \\
\hline MMCD & 5.376 & 79 & $32,71( \pm 18,75)$ & 168,35 \\
\hline MMCD-Anti-TNF ( \pm MMCD ou MTX) & 1.087 & 16 & $41,80( \pm 19,12)$ & $1.809,04$ \\
\hline MMCD-MTX & 374 & 5 & $36,01( \pm 19,52)$ & 92,21 \\
\hline Iniciaram com Imuno & 89 & 1 & $37,34( \pm 22,11)$ & 616,91 \\
\hline IMUNO & 48 & 54 & $29,26( \pm 19,66)$ & 132,55 \\
\hline IMUNO-Anti-TNF ( \pm MMCD ou MTX) & 21 & 24 & $46,01( \pm 19,78)$ & $2.174,37$ \\
\hline IMUNO-MMCD ou MTX & 20 & 22 & $47,65( \pm 23,14)$ & 144,02 \\
\hline OUTROS & 1.156 & 10 & $42,26( \pm 17,74)$ & 641,96 \\
\hline Total & 11.573 & 100 & $33,52( \pm 18,70)$ & 938,33 \\
\hline
\end{tabular}

ADA = Adalimumabe; ETA = Etanercepte; INF = Infliximabe; IMUNO = Imunossupressores; $\mathrm{MMCD}=$ Medicamentos Modificadores do Curso da Doença; Anti-TNF = Anti-tumor necrosis fator; MTX = Metotrexato. 
Tabela 4. Análise multivariada do gasto médio mensal ambulatorial dos usuários do Sistema Único de Saúde de Minas Gerais que utilizaram medicamentos para tratamento da Artrite Reumatóide (janeiro de 2008 a setembro de 2013)

\begin{tabular}{|c|c|c|c|}
\hline Variável dependente & & & \\
\hline \multicolumn{4}{|l|}{ Gasto médio mensal } \\
\hline Variável explicativa & $\boldsymbol{\beta}$ & Erro Padrão & $\mathbf{p}$ \\
\hline Intercepto & 225,56 & 56,98 & $<0,001$ \\
\hline Idade & $-3,12$ & 0,56 & $<0,001$ \\
\hline \multicolumn{4}{|l|}{ Sexo } \\
\hline Feminino/Masculino & 27,86 & 19,88 & $>0,05$ \\
\hline Tempo de acompanhamento (meses) & 6,90 & 0,71 & $<0,001$ \\
\hline \multicolumn{4}{|l|}{ Ano de entrada na coorte } \\
\hline $2009 / 2008$ & 92,39 & 30,10 & $<0,05$ \\
\hline $2010 / 2008$ & 198,91 & 33,62 & $<0,001$ \\
\hline $2011 / 2008$ & 237,01 & 30,23 & $<0,001$ \\
\hline $2012 / 2008$ & 202,96 & 38,74 & $<0,001$ \\
\hline $2013 / 2008$ & 101,31 & 54,32 & $>0,05$ \\
\hline \multicolumn{4}{|l|}{$\mathrm{CID}^{*}$} \\
\hline $\begin{array}{l}\text { Síndrome de Felty (M05.0)/Outras artrites reumatóides soro-positivas } \\
\text { (M05.8) }\end{array}$ & 19,79 & 20,18 & $>0,05$ \\
\hline $\begin{array}{l}\text { Artrite reumatóide soro-negativa (M06.0)/Outras artrites reumatóides } \\
\text { soro-positivas (M05.8) }\end{array}$ & $-75,08$ & 22,97 & 0,001 \\
\hline $\begin{array}{l}\text { Artrite reumatóide com comprometimento de outros órgãos e sistemas } \\
\text { (M05.3)/Outras artrites reumatóides soro-positivas (M05.8) }\end{array}$ & 9,63 & 30,19 & $>0,05$ \\
\hline $\begin{array}{l}\text { Outras artrites reumatóides especificadas (M06.8)/Outras artrites } \\
\text { reumatóides soro-positivas (M05.8) }\end{array}$ & $-24,48$ & 37,83 & $>0,05$ \\
\hline $\begin{array}{l}\text { Artrite reumatóide juvenil (M08.0)/Outras artrites reumatóides soro- } \\
\text { positivas (M05.8) }\end{array}$ & $-248,74$ & 63,57 & $<0,001$ \\
\hline $\begin{array}{l}\text { Doença reumatóide do pulmão (M05.1)/Outras artrites reumatóides } \\
\text { soro-positivas (M05.8) }\end{array}$ & $-38,91$ & 72,05 & $>0,05$ \\
\hline $\begin{array}{l}\text { Vasculite reumatóide (M05.2)/Outras artrites reumatóides soro- } \\
\text { positivas (M05.8) }\end{array}$ & $-233,00$ & 122,43 & $<0,05$ \\
\hline \multicolumn{4}{|l|}{ Esquema Terapêutico* } \\
\hline MTX/MMCD & $-219,19$ & 23,30 & $<0,001$ \\
\hline $\mathrm{ADA} / \mathrm{MMCD}$ & $1.915,46$ & 28,82 & $<0,001$ \\
\hline ETA/MMCD & $4.012,50$ & 30,52 & $<0,001$ \\
\hline INF/MMCD & $1.609,61$ & 44,42 & $<0,001$ \\
\hline IMUNO/MMCD & 259,28 & 79,23 & 0,001 \\
\hline \multicolumn{4}{|l|}{ IDH-M do município de residência* } \\
\hline $0,78-0,80 / 0,00-0,77$ & 25,21 & 21,23 & $>0,05$ \\
\hline $0,81-0,83 / 0,00-0,77$ & 51,59 & 22,47 & $<0,05$ \\
\hline 0,84 ou mais $/ 0,00-0,77$ & 3,00 & 22,84 & $>0,05$ \\
\hline
\end{tabular}

do GMM $\left(\mathrm{R}^{2}=66,72 \%\right)$. Este é menor com o aumento da idade dos pacientes $(\mathrm{p}<0,001)$ e quando estes são do sexo masculino $(\mathrm{p}>0,05)$.
O tempo de acompanhamento também parece impactar no GMM, que aumenta com o aumento do tempo de acompanhamento $(\mathrm{p}<0,001)$, 
assim como ocorre em relação ao ano de entrada na coorte, não sendo estatisticamente significativo apenas para o ano de 2013.

Em comparação ao CID de registro mais frequente entre os pacientes da coorte, "Outras artrites reumatoides soro-positivas", apenas os CID "Artrite reumatoide soro-negativas" ( $p<0,001)$, "Artrite reumatoide juvenil" ( $\mathrm{p}<0,001)$ e Vasculite reumatoide $(\mathrm{p}<0,05)$ impactaram de forma estatisticamente significativa no GMM. Pacientes com estes diagnósticos registrados no início do acompanhamento apresentaram tendência de menor gasto.

A tendência de menor gasto para pacientes em uso de MTX no início do acompanhamento também foi identificada nesta análise $(\mathrm{p}<0,001)$. Pacientes que residiam em municípios de maior IDH-M apresentaram maior GMM, sendo este resultado estatisticamente significativo apenas para a faixa $0,81-0,83(\mathrm{p}<0,05)$. A análise de resíduos e o teste de Breusch-Pagan studentizado demonstrou que o modelo final é adequado para analisar a relação linear entre o gasto médio mensal e as variáveis incluídas $(\mathrm{p}<0,001)$.

\section{Discussão}

Neste estudo observou-se que a maioria dos pacientes era mulher e tinha idade entre 37 e 67 anos, com média de idade de 52 anos. No Brasil, o perfil dos pacientes em relação ao sexo e idade não é diferente do observado para a coorte de $M^{16-23}$. Estudos internacionais incluindo pacientes com AR apresentaram achados semelhantes ${ }^{24-32}$. Os motivos para maior frequência da doença em mulheres podem estar relacionados à possível influência hormonal no processo inflamatório causador da $\mathrm{AR}^{18,31-33}$, bem como ao maior percentual de utilização dos serviços de saúde por pessoas do sexo feminino ${ }^{34}$.

Dentre os diagnósticos mais frequentes observados na coorte, o "Síndrome de Felty" é o que chama mais atenção. Apesar deste diagnóstico ser uma condição de baixa prevalência entre os pacientes acometidos por $\mathrm{AR}^{35}$, o mesmo foi identificado para $25 \%$ dos indivíduos da coorte de MG. Este diagnóstico também foi observado entre os mais frequentes em Costa et al. ${ }^{10}$, sendo que os autores atribuem essa alta prevalência a dois fatores principais: limitações de utilização de banco de dados administrativos que podem sofrer erros no registro das informações e dificuldade em se determinar a Síndrome de Felty, que pode ser confundida com a "pseudo-síndrome de
Felty", com prevalência duas vezes maior que a primeira $^{10}$.

O tratamento da AR varia de acordo com o estágio, a atividade e a gravidade da doença e tem por objetivos melhorar a qualidade de vida dos pacientes, controlar a progressão das lesões articulares, prevenir a perda funcional e diminuir a dor ${ }^{19,24,31}$. Abreu et al. ${ }^{16}$ relataram que, no período de 1979 a 1994, o tratamento predominante para artrite reumatoide incluía o uso de anti-inflamatórios não esteroidais (AINES), seguido do uso de corticoides. Os autores observaram que ao longo dos anos estes tratamentos entraram em desuso dando lugar aos MMCD sintéticos, principalmente ao MTX ${ }^{16}$.

A introdução dos MMCD biológicos no mercado mundial se deu em 1998, com a aprovação do ETA nos Estados Unidos ${ }^{18,29}$. No Brasil, estes medicamentos passaram a ser disponibilizados pelo SUS em 2006, com a publicação do PCDT $^{2}$ para o tratamento da AR. Este último, em consonância com as diretrizes para o tratamento da $\mathrm{AR}^{36}$, recomenda o início do tratamento com MTX e, em caso de contraindicação, pode-se utilizar outros MMCD sintéticos². Para os pacientes com menor risco de mau prognóstico, o tratamento com antimaláricos passa a ser a primeira escolha e, em caso de falha com o MMCD em monoterapia, a associação entre eles é indicad ${ }^{36}$. O início da terapia com os MMCD biológicos é indicado após seis meses de terapia com MMCD sintéticos e persistência de atividade doença moderada a alta $^{36}$. Recomenda-se a associação destes medicamentos aos MMCD sintéticos e, apenas em caso de contraindicação absoluta a estes últimos, utilizar MMCD biológicos em monoterapia $^{36}$. Em caso de falha com MMCD biológico inicial, pode-se trocar por outro biológico ${ }^{36}$.

No presente estudo, a maioria dos pacientes iniciou o acompanhamento em tratamento com MTX ou outros MMCD. Torna-se necessário ressaltar que, por se tratar de um estudo cuja fonte de dados é administrativa, os achados podem não refletir em plenitude a prática clínica. Considerando que o ponto de corte de início de acompanhamento da coorte é janeiro 2008, o tratamento experimentado pelos indivíduos antes desta data não foi observado. Este fato pode explicar $\mathrm{o}$ alto percentual de indivíduos que iniciaram o tratamento com ANTI-TNF. Estudo realizado no Paraná, que avaliou apenas pacientes em uso de Anti-TNF, em que a fonte de dados também foi administrativa, observou que $44 \%$ dos pacientes receberam tratamento com ETA, $40 \%$ com ADA e $16 \%$ com INF ${ }^{22}$. 
Assim como no presente estudo, em Florianópolis/SC (2010) foi observado que a maioria dos pacientes iniciou o tratamento com MMCD associados e ao longo do tratamento passaram a utilizar Anti-TNF em monoterapia ou MMCD em monoterapia ${ }^{18}$. De acordo com o Registro Brasileiro de Biológicos (BIOBADA BRASIL), no período entre 2008 e 2012, 44\% dos pacientes foram tratados com INF, 29\% com ADA e $27 \%$ com ETA. Desses, 43\% estavam em uso associado com MMCD e $30 \%$ com corticosteroides ${ }^{37}$. Estudo multicêntrico realizado em países do Centro e do Leste da Europa mostrou que o acesso a medicamentos biológicos para o tratamento da AR variou entre 1,0 a 5,0\% dos pacientes ${ }^{32}$. Segundo os autores, o uso destes medicamentos em diferentes países reflete preferências e prioridades dos mesmos ${ }^{32}$.

O tempo médio de permanência dos indivíduos na coorte de MG foi de 33,5 $( \pm 18,7)$ meses e variou conforme o seguimento terapêutico. Observou-se maior tempo médio de acompanhamento para os casos de associação ou troca do Anti-TNF. Este achado pode estar relacionado ao fato destes medicamentos se diferenciarem dos MMCD clássicos por sua especificidade contra alvos terapêuticos definidos, o que lhes fornece características importantes para o tratamento da AR como rápido início de ação, resposta sustentada e maior tolerabilidade ${ }^{19}$.

Pacientes que iniciaram com INF trocaram de Anti-TNF em maior frequência do que aqueles que iniciaram com ADA ou ETA. A troca de um Anti-TNF por outro pode ser uma opção terapêutica em casos de ineficácia ou intolerância ao primeiro, uma vez que, apesar de apresentarem eficácia semelhante, não são idênticos nos aspectos de estrutura molecular, farmacocinética, mecanismo de ação, potencial de formação de autoanticorpos e anticorpos humanos anti-quiméricos ou anti-humanos, indução de apoptose e posologia ${ }^{38}$. Sendo o presente estudo fruto de análise de um banco de dados administrativos, é carente de dados relacionados ao estado clínico do paciente e, por isso, não foi possível avaliar os fatores que influenciam a troca de esquema terapêutico na coorte de MG.

$\mathrm{O}$ avanço do tratamento da AR com a inclusão dos Anti-TNF é reconhecido. Entretanto, os custos das novas tecnologias empregadas devem ser considerados antes da incorporação e durante seu uso para avaliação de sua manutenção nos protocolos de tratamento estabelecidos. Segundo Carias et $\mathrm{al}^{39}$, os gastos com o CBAF aumentaram cerca de $159 \%$ no período de 2003 a 2006.
Ainda segundo os autores, em 2007, o gasto com medicamentos especializados chegou à esfera de $\mathrm{R} \$ 2,3$ bilhões e os medicamentos para AR representaram 10,4\% deste valor ${ }^{39}$.

Em MG, os esquemas terapêuticos de maior GMM por paciente foram aqueles que incluíam o uso dos Anti-TNF. Para fins de comparação, os valores de gastos/custos de estudos nacionais citados na sequência dessa discussão foram também atualizados pelo IPCA para valores de dezembro de 2015. Achados semelhantes foram observados por Wiens et al. ${ }^{22}$, ao considerar os dados de dispensação de medicamentos de alto custo registrados no sistema informatizado do Paraná em março de 2010. Bagatini ${ }^{18}$ apontou para um custo direto anual com a assistência prestada ao paciente com AR de $\mathrm{R} \$ 30.223,67$ por paciente/ano. Deste valor, 70,4\% foram financiados pelo SUS e os medicamentos contribuíram com $91,4 \%$ para sua composição ${ }^{18}$. Foram considerados custos diretos médicos pelos autores os custos com medicação essencial e secundária, administração de medicamento parenterais, consultas médicas, urgências médicas, terapias e atividades físicas, exames complementares e hospitalizações ${ }^{18}$. Em Florianópolis, o custo direto médico estimado para o tratamento da AR por paciente foi de $\mathrm{R} \$ 28.097,85$, sendo observada diferenças significativas nos custos diretos para pacientes tratados com MMCD (R\$ 6.259,09) e aqueles tratados com Anti-TNF (R $\$ 59.961,07)^{18}$.

Em estudo realizado por Venson et al. ${ }^{40}$, foram identificados os seguintes custos anuais para o tratamento da AR, no ano de 2008: MTX = R\$ 183,$08 ; \mathrm{INF}=\mathrm{R} \$ 25.652,84 ; \mathrm{ADA}=\mathrm{R} \$ 29.916,67$; $\mathrm{ETA}=\mathrm{R} \$ 37.055,54$. Em 2012, o Ministério da Saúde identificou que o medicamento de maior gasto anual era o ETA ( $\mathrm{R} \$ 37.170,30)$, seguido do ADA (R\$36.721,66) e INF (R\$25.721,27) ${ }^{20}$. Segundo Lundkvist et al. ${ }^{41}$, o custo médio anual por paciente varia conforme o protocolo adotado em cada país, bem como de acordo com a metodologia adotada por cada estudo para estimá-lo. Em um estudo multicêntrico realizado em países da Europa central e oriental, também foram observadas diferenças para o custo anual por paciente ${ }^{32}$.

Para a coorte de MG avaliou-se, também, as variáveis que poderiam explicar o GMM no tratamento da AR. A análise múltipla apontou para uma relação negativa entre idade e maior gasto médio mensal após o controle pelas outras variáveis $(p<0,001)$. Uma possível explicação pode ser o fato de que a média de idade entre os indivíduos que iniciaram o acompanhamento com MMCD foi maior do que entre aqueles que iniciaram o 
acompanhamento com algum Anti-TNF ( $\mathrm{p}<$ 0,0001 ), considerando que o tratamento com estes últimos é significativamente mais caro do que o tratamento com MMCD. Michaud et al. $.^{28} \mathrm{ob}-$ servaram perfil semelhante em estudo realizado nos Estados Unidos onde ocorreu redução anual do gasto com o tratamento da AR, com o aumento da idade, para indivíduos acima de 65 anos.

O tempo também foi fator determinante para o aumento do GMM ao longo do acompanhamento ( $p<0,001)$. Achados de uma análise econômica do tratamento da AR apontaram para uma progressão de custo que ocorre ao longo do tempo de tratamento devido à substituição de MMCD mais antigos pelos mais novos, que são mais $\operatorname{caros}^{42}$. Na coorte na coorte de MG, indivíduos em idade superior a 70 anos apresentaram menor tempo médio de acompanhamento, 29,91 $( \pm 17,25)$ meses $(\mathrm{p}<0,001)$. Este achado também pode explicar a redução do GMM com o avançar da idade. Relação positiva entre o sexo feminino e o maior GMM com o tratamento da AR também foi encontrada. Esse resultado é coerente com a maior frequência da doença em mulheres e a maior utilização de serviços de saúde por elas ${ }^{34}$. Estudos demonstram que o custo da doença pode variar de acordo com o tempo de doença, com sua evolução e com o tipo de esquema terapêutico utilizado ${ }^{25,28,32}$.

Para a avaliação do perfil sociodemográfico dos participantes da coorte, utilizou-se como indicador o IDH-M. Segundo o Programa das Nações Unidas para o Desenvolvimento (PNUD), quanto maior o IDH, maior o desenvolvimento nas áreas: renda, educação e saúde ${ }^{42}$. Em MG, os pacientes eram residentes em municípios que estavam distribuídos proporcionalmente entre as faixas de IDH-M e observou-se relação positiva entre o maior IDH-M do município de residência do indivíduo e o aumento GMM. Este resultado pode estar relacionado com o fato de locais mais desenvolvidos economicamente possuírem maior acesso aos serviços de saúde e, principalmente, maior concentração de reumatologistas ${ }^{10,22,43,44}$.

Estudo realizado por Travassos et al. ${ }^{43} \mathrm{de}-$ monstrou que o acesso aos serviços de saúde no Brasil é influenciado pela condição social dos indivíduos, bem como pelo local de residência. Para este último fator, o estudo indicou que o acesso aos serviços de saúde melhora com o grau de desenvolvimento socioeconômico da região de residência ${ }^{43}$. Wiens et al. ${ }^{22}$ corroboram com esses achados ao observar que metade dos brasileiros com $\mathrm{AR}$ não possuem diagnóstico estabelecido e as principais barreiras para o acesso ao tratamento da doença são: baixa disponibilidade de reumatologistas, dificuldades de acesso da população aos medicamentos e demora no agendamento de consultas médicas.

Observou-se significância estatística para a relação negativa entre alguns diagnósticos identificados à entrada na coorte de MG e o GMM. Um estudo europeu estimou que os custos diretos para cada paciente aumentam, por ano, duas vezes mais para aqueles com alta atividade da doença quando comparados com aqueles com baixa atividade da doença ${ }^{41}$. Em relação ao esquema terapêutico adotado, aqueles que incluíam os medicamentos Anti-TNF possuem maior GMM em comparação com aqueles que incluíam outros MMCD ( $\mathrm{p}<0,001)$. Monteiro e Zanini ${ }^{11}$ encontraram resultados semelhantes ao avaliar os custos dos diferentes ciclos de tratamento elaborados a partir das recomendações do protocolo clínico para tratamento da AR da Sociedade Brasileira de Reumatologia. Na Alemanha ${ }^{45}$ e na Espanha $^{24}$ foram observados resultados similares.

\section{Limitações do método}

Os dados foram extraídos de banco de dados administrativos que têm por finalidade a gestão financeira do CEAF e que são alimentadas por informações fornecidas pelas Secretarias de Estado da Saúde. Portanto, a qualidade desta informação é dependente dos processos envolvidos no registro e na disponibilização dos dados por sistemas de informação. Sendo assim, é possível a existência de dados incorretos causados por erros na alimentação das bases de dados ou de sub-registros que podem culminar em sub ou superestimação das análises realizadas.

Outra limitação importante do presente estudo refere-se ao fato de que os gastos analisados abrangem apenas aqueles relacionados aos valores de medicamentos registrados no SIA/SUS, que representam os valores reembolsados pelo Ministério da Saúde e não os valores reais dos recursos de saúde utilizados. Por não incluir outros gastos ambulatoriais como, por exemplo, gastos despendidos para infusão do INF, podem subestimar o custo real do tratamento da Artrite Reumatoide.

\section{Conclusão}

A avaliação na coorte de Minas Gerais, a partir da construção de sua linha de cuidado, por meio do pareamento probabilístico de base dados administrativos, permitiu identificar alguns fatores 
que podem impactar no gasto com o tratamento medicamentoso da AR, uma doença crônica com importante impacto econômico para o SUS.

Observou-se que este gasto é influenciado por características como sexo, idade dos pacientes, tempo de acompanhamento e condições sociodemográficas. Verificou-se que o nível de desenvolvimento dos municípios onde residem estes pacientes atua de forma negativa no gasto com o tratamento da AR e este fato pode estar relacionado ao acesso aos serviços de saúde, à consulta com reumatologistas e, consequentemente, ao acesso a terapias mais avançadas. Apesar dos desafios apresentados para a garantia do cuidado ao paciente portador de AR, a coorte de Minas Gerais apontou para o cumprimento do protocolo de tratamento proposto pelo Ministério da Saúde e que este protocolo mostra coerência com protocolos estabelecidos em outros países.

Cabe ressaltar que os estudos nacionais publicados que abordam os custos com o tratamento da Artrite Reumatoide são, em sua maioria, de descrição da composição dos gastos e/ou custo registrados em bases administrativas do SUS. Desta forma, geralmente não abordam os fatores que podem influenciar na variabilidade dos mesmos. Neste sentido, a maior contribuição desse estudo é a proposição de uma metodologia que demonstra a possibilidade de, a partir de bancos de dados administrativos, realizar avaliações de custos/gastos das linhas de cuidado adotadas no SUS, bem como dos principais fatores que podem influenciar sua variabilidade. Este fato é de grande relevância ao considerarmos que, no Brasil, não é usual o desenvolvimento de sistemas de informação que têm a finalidade de coletar dados clínicos para utilização na gestão do sistema de saúde. Sistemas de informação que possibilitam extrair tanto dados clínicos quanto de custos/gastos podem ser ferramenta importantes na construção de estudos econômicos em saúde que permitam subsidiar as avaliações de políticas públicas de saúde, tanto do ponto de vista clínico como do ponto de vista da gestão.

\section{Colaboradores}

GD Silva trabalhou na concepção, no delineamento, na análise e interpretação dos dados. Redigiu o artigo, realizou a revisão crítica e aprovou a versão a ser publicada. EIG Andrade trabalhou na concepção dos dados e realizou a revisão crítica e aprovou a versão a ser publicada. ML Cherchiglia trabalhou na concepção dos dados e realizou a revisão crítica e aprovou a versão a ser publicada. AA Guerra Júnior trabalhou na concepção e no delineamento dos dados. Realizou a revisão crítica e aprovou a versão a ser publicada. AM Almeida trabalhou na concepção e no delineamento dos dados. Realizou a revisão crítica da versão a ser publicada. FA Acurcio trabalhou na concepção, no delineamento, na análise e interpretação dos dados. Participou da redação do artigo, realizou a revisão crítica e aprovou a versão a ser publicada.

\section{Agradecimentos}

Ao apoio financeiro do Conselho Nacional de Desenvolvimento Científico e Tecnológico CNPq e Fundação de Amparo à Pesquisa do Estado de Minas Gerais - FAPEMIG. 


\section{Referências}

1. Mota LMH, Cruz BA, Brenol CV, Pereira IA, Fronzas LSR, Bertolo MB, Freitas MVC, Silva NA, Louzada-Junior P, Giorgi RDN, Lima RAC, Pinheiro GRC. Consenso da Sociedade Brasileira de Reumatologia 2011 para o diagnóstico e avaliação inicial da artrite reumatoide. Rev Bras Reumatol 2011; 51(3):207-219.

2. Brasil. Ministério da Saúde (MS). Portaria SCTIE/MS $\mathrm{n}^{\circ} 66$ de 06 de novembro de 2006. Aprova o protocolo clínico e diretrizes terapêuticas: artrite reumatoide. $\mathrm{Di}$ ário Oficial da União 2006; 06 nov.

3. Mota LMH, Laurindo IMM, Neto LLS. Princípios Gerais do tratamento da artrite reumatoide inicial. Revista da Associação Médica Brasileira 2010; 56(3):360-362.

4. Marques-Neto JF, Gonçalves ET, Langen LFOB, Cunha MFL, Radominski S, Oliveira SM, Cury SE, Medeiros F, Sampaio GC. Estudo multicêntrico da prevalência da artrite reumatoide do adulto em amostras da população brasileira. Rev Bras Reumatol 1993; 33(5):169-173.

5. Senna ER, Barros AL, Silva EO, Costa IF, Pereira LV, Ciconelli RM, Ferraz MB. Prevalence of rheumatic diseases in Brazil: a study using the COPCORD approach. J Rheumatol 2004; 31(3):594-597.

6. Alamanos Y, Voulgari PV, Drosos AA. Incidence and Prevalence of Rheumatoid Arthritis, Based on the 1987 American College of Rheumatology Criteria: A Systematic Review. Semin Arthritis Rheum 2006; 36(3):182-188.

7. Furst DE, Schiff MH, Fleishmann RM, Strand V, Birbara CA, Compagagnone D, Fischkoff SA, Chartash EK. Adalimumabe, a fully anti tumor necrosis factor-alpha monoclonal antibody, and concomitant standard antirheumatic therapy for the treatment or rheumatoid arthritis: results of STAR (Safety Trial of AdalimumB IN Rheumatoid atthritis). J Rheumatol 2003; 30(12):25632571.

8. Brasil. Ministério da Saúde (MS). Assistência farmacêutica: instruções técnicas para sua organização. Brasília: MS; 2001.

9. Brandão CMR, Guerra Junior AA, Cherchiglia ML, Andrade EIG, Almeida AM, Silva GD, Queiroz OV, Faleiros DR, Acurcio FA. Gastos do Ministério da Saúde do Brasil com Medicamentos de Alto Custo: Uma Análise Centrada no Paciente. Value in Health 2011; 14(5 Supl.):S71-S77.

10. Costa JO, Almeida AM, Júnior AAG, Cherchiglia ML, Andrade EIG, Acurcio FA. Tratamento da artrite reumatoide no Sistema Único de Saúde, Brasil: gastos com Infliximabe em comparação com medicamentos modificadores do curso da doença sintéticos, 2003 a 2006. Cad Saude Publica 2014; 30(2):283-295.

11. Monteiro RDC, Zanini AC. Análise de custo do tratamento medicamentoso da artrite reumatoide. Revista Brasileira de Ciências Farmacêuticas 2008; 44(1):25-33.

12. Conselho Nacional de Secretários da Saúde (CONASS). Para entender a gestão do programa nacional de dispensação em caráter excepcional. Brasília: CONASS; 2004.

13. Cherchiglia ML, Júnior AFG, Andrade EIG, Machado CJ, Acúrcio FA, Meira Junior W, Paula BD, Queiroz OV. A construção da base de dados nacional em Terapia Renal Substitutiva (TRS) centrada no indivíduo: aplicação do método de linkage determinístico probabilístico. Revista Brasileira de Estudos de População 2007; 24(1):163-167.
14. Christen P, Churches T, Hegland M. A parallel open source data linkage system. [acessado 2016 Mar 6]. Disponível em: http://citeseer.ist.psu.edu/christen04parallel.html

15. Instituto Brasileiro de Geografia e Estatística (IBGE). Indice de Desenvolvimento Humano Municipal IDH-M. [acessado 2016 Mar 6]. Disponível em: http:// cidades.ibge.gov.br/cartograma/mapa.php?lang=\&coduf $=31 \& \operatorname{codmun}=310620 \&$ idtema $=118 \& \operatorname{codv}=\mathrm{v} 01 \&$ search=minas-gerais|belo-horizonte|sintese-das-informacoes-

16. Abreu MM, Kowalski SC, Ciconelli RM, Ferraz MB. Avaliação do perfil sócio-demográfico, clínico-laboratorial e terapêutico dos pacientes com artrite reumatoide que participaram de projetos de pesquisa na Escola Paulista de Medicina, nos últimos 25 anos. Rev Bras Reumatol. 2006; 46(2):103-109.

17. Cavalcanti MP. Levantamento estatístico dos pacientes atendidos pela ADORE (Associação dos Portadores de Doença Reumática) [monografia]. Curitiba: Universidade Tuiuti do Paraná; 2006.

18. Bagatini F. Estimativa dos custos relacionados à artrite reumatoide em pacientes atendidos por meio do Componente Especializado da Assistência Farmacêutica/MS na Farmácia Escola UFSC/PMF, 2008-2010 [dissertação]. Florianópolis: Universidade Federal de Santa Catarina; 2010.

19. Faleiro LR, Araújo LHR, Varavallo MA. A terapia Anti -TNF- na artrite reumatoide. The Anti-TNF- therapy in the rheumatoid arthritis. Semina 2011; 32(1):77-94.

20. Brasil. Ministério da Saúde (MS). Comissão Nacional de Incorporação de Tecnologias no SUS - CONITEC. Medicamentos biológicos (Infliximabe, etanercepte, adalimumabe, rituximabe, abatacepte, tocilizumabe, golimumabe e certolizumabe pegol) para o tratamento da Artrite Reumatoide. Brasília: Secretaria de Ciência, Tecnologia e Insumos Estratégicos; 2012 Jun. Relatório de Recomendação da Comissão Nacional de Incorporação de Tecnologias no SUS - CONITEC. [citado 27 Jan 2013]. Disponível em: http://formsus.datasus.gov.br/ imgarq/8793/1101992_109700.pdf

21. Louzada-Junior P, Souza BDB, Toledo RA, Ciconelli RM. Análise descritiva das características demográficas e clínicas de pacientes com artrite reumatoide no estado de São Paulo, Brasil. Rev Bras Reumatol 2007; 47(2):84-90.

22. Wiens A, Grochocki MC, Pontarolli DRS, Venson R, Correr CJ, Pontarolo R. Perfil dos usuários de anticitocinas disponibilizadas pelo Sistema Único de Saúde no estado do Paraná para o tratamento da artrite reumatoide. Revista Brasileira de Reumatologia 2012; 52(2):203-213.

23. Buendgens FB, Blatt CR, Marasciulo ACE, Leite SN, Farias MR. Estudo de custo-análise do tratamento da artrite reumatoide grave em um município do sul do Brasil. Cad Saude Publica 2013; 29(Supl.):S81-S91.

24. Varela AM, Rodriguez JB, Freire AC. Aproximación al coste del tratamento farmacológico em la artritis reumatoide em España. An Med Interna (Madrid) 2003; 3(20):114-121. 
25. Ruof J, Hulsemann JL, Mittendorf T, Handelmann S, van der Schulenburg JM, Zeidler H, Merkesdal S. Costs of rheumatoid arthritis in Germany: a micro-costing approach based on healthcare payer's data sources. Ann Rheum Dis 2003; 62(6):544-550.

26. Kolbelt G, Eberhardt K, Geborek P. TNF inhibitors in the treatment of rheumatoid arthritis in clinical practice: cost and outcomes in a follow up study of patients with RA treated with etanercept or infliximab in southern Sweden. Ann Rheum Dis 2004; 63(1):4-10.

27. Hallert E, Husberg M, Skogh T. 28-joint count disease activity score at 3 months after diagnosis or early rheumatoid arthritis is strongly associated with direct and indirect costs over the following 4 years: the Swedish TIRA project. Rheumatology 2011; 50(7):1259-1267.

28. Michauad K. Messe J, Choi HK, Wolfe F. Direct medical cost and their predictors in patients with rheumatoid arthritis. Arthritis \& Rheumatism 2003; 48(10):27502762 .

29. Birnbaum HG, Pike C, Banerjee R, Waldman T, Cifaldi M. Changes in utilization and costs for patients with rheumatoid arthritis, 1997 to 2006. Pharmacoeconomics 2012; 30(4):323-336.

30. Kvamme MK, Lie E, Kvien TK, Kristiansen IS. Two-year direct and indirect costs for patients with inflammatory rheumatic joint diseases: data from real-life follow-up or patients in the NOR-DMARD registry. Rheumatology 2012; 51(9):1618-1627.

31. Schneiders RE. Caracterização dos gastos do Ministério da Saúde com medicamentos para Artrite Reumatoide, no âmbito do Componente Especializado da Assistência Farmacêutica, no período de 2008 a 2009 [dissertação]. Brasília: Universidade de Brasília; 2012.

32. Orlewska E, Ancuta I, Anic B, Codrenau C, Damjanov N, Djukic P, Ionescu R, Marinchev L, Nasonov EL, Peets T, Praprotnik S, Rashkov R, Skoupa J, Tlustochowicz W, Tlustochowicz M, Tomsic M, Veldi T, Vojinovic J, Wiland P. Acess to biologic treatment for rheumatoid arthritis in Central and Eastern European (CEE) countries. Med Sci Moint 2011; 17(4):SR1-13.

33. Alamanos Y, Drosos AA. Epidemiology of adult rheumatoid arthritis. Autoimmunity Reviews 2005; 4(3):130-136

34. Intituto Brasileiro de Geografia e Estatística (IBGE). Pesquisa nacional de saúde 2013: acesso e utilização dos serviços de saúde, acidentes e violências - Brasil, grandes regiões e unidades da federação. Rio de Janeiro: IBGE; 2015.

35. Rosenstein ED, N Kramer. Felty's and Pseudo-Felty's Syndromes. Seminars in Arthritis and Rheumatism 1991; 21(3):129-142.

36. Brasil. Ministério da Saúde (MS). Portaria nº710, de 27 de junho de 2013. Aprova o Protocolo Clínico e Diretrizes Terapêuticas da Artrite Reumatoide. Diário Oficial da União 2013; 27 jun.
37. Fafá BP, Louzada-Junior P, Titton DC, Zandonade E, Ranza R, Laurindo I, Peçanha P, Ranzolin A, Hayata AL, Duarte A, Silveira IG, Costa I, Macieira JC, Guedes-Barbosa LS, Bertolo MB, Sauma MF, Silva MB, Freire M, Scheinberg MA, Fernandes V, Bianchi W, Miranda JR, Pinheiro GR, Carvalho HM, Brenol CV, Pereira IA, de Castro GR, de Morais JC, Oliveira SK, de Abreu MM, Toledo RA, Pinheiro MM, Vieira WP, Valim V; BIOBADABRASIL. Drug survival and causes of discontinuation of the first anti-TNF in ankylosing spondylitis compared with rheumatoid arthritis: analysis from BIOBADABRASIL. Clin Rheumatol 2015; 34(5):921-927.

38. Alonso-Ruiz A, Pijoan JI, Ansuategui E, Urkaregi A, Calabozo M, Quintana A. Tumor Necrosis fator alpha in rheumatoid arthritis: systematic rewew and mataanalysis of efficacy and safety. BMC Muscoloskeletal Disorders 2008; 9(52):1-27.

39. Carias CM, Vieira FS, Giordano CV, Zucchi P. Medicamentos de dispensação excepcional: histórico e gastos do Ministério da Saúde do Brasil. Rev Saude Publica 2011; 45(2):233-240.

40. Venson R, Wiens A, Correr CJ, Otuki MF, Grochocki MC, Pontarolli DRS, Pontatolo R. Avaliação econômica das anticitocinas: adalimumabe, etanercepte e Infliximabe no tratamento da artrite reumatoide no Estado do Pará. Revista de Saúde Coletiva 2011; 21(2):359-376.

41. Lundkvist J, Kastang F, Kolbelt G. The Burden of rheumatoid arthrists and access to treatment: health burden an cost. Eur J Health Econ 2008; 8(Supl. 2):S49-S60.

42. Programa das Nações Unidas para o Desenvolvimento (PNUD). [Internet]. Brasil: Ranking do IDH dos Municípios do Brasil 2003. [citado 27 Jan 2012]. Disponível em: http://www.pnud.org.br/atlas/ranking/IDH_Municipios_Brasil_2000.aspx?indiceAccordion=1\&li=li_ Ranking2003

43. Travassos C, Oliveira EXG, Viacava F. Desigualdades geográficas e sociais no acesso aos serviços de saúde no Brasil: 1998 e 2003. Cien Saude Colet 2006; 11(4):975986.

44. Albuquerque CP. Inequalidade na distribuição de reumatologistas no Brasil: correlação com local de residência médica, Produto Interno Bruto e Índice de Desenvolvimento Humano. Rev Bras Reumatol 2014; 54(3):166-171.

45. Verstappen SMM, Verkleij H, Bijlsma JWJ, Buskens E, Kruize AA, Heurkens AHM, Van Der Veen MJ, Jacobs JW. Determinants of direct cost in Dutch rheumatoid arthritis patients. Ann Rheum Dis 2004; 63(7):817-824.
Artigo apresentado em 16/11/2015

Aprovado em 01/07/2016

Versão final apresentada em 03/07/2016 